\title{
Whole-Transcriptome Analysis of CD133 + CD144+ Cancer Stem Cells Derived from Human Laryngeal Squamous Cell Carcinoma Cells
}

\author{
Yongyan Wu $\mathrm{u}^{\mathrm{a}, \mathrm{b}, \mathrm{c}, \mathrm{d}} \quad$ Yuliang Zhang ${ }^{\mathrm{a}, \mathrm{b}, \mathrm{c}, \mathrm{d}}$ Min Niu $\mathrm{u}^{\mathrm{a}, \mathrm{b}, \mathrm{c}, \mathrm{d}} \quad$ Yong Shi $\mathrm{i}^{\mathrm{a}, \mathrm{b}, \mathrm{c}, \mathrm{d}}$ \\ Hongliang Liu ${ }^{a, b, c, d}$ Dongli Yang ${ }^{a, b, c, d}$ Fei Li $i^{a, b, c, d}$ Yan Lue Yunfeng Bo ${ }^{f}$ \\ Ruiping Zhang ${ }^{d, g}$ Zhenyu Lid, hongjie Luo ${ }^{a, b, c, c, d}$ Jiajia Cuja,b,c,d \\ Jiangwei Sang ${ }^{a, b, c, d} \quad$ Caixia Xiang ${ }^{a, b, c, d}$ Wei Gao ${ }^{a, b, c, d}$ Shuxin Wen ${ }^{a, b, c, d}$
}

aShanxi Key Laboratory of Otorhinolaryngology Head and Neck Cancer, Taiyuan, bDepartment of Otolaryngology Head \& Neck Surgery, The First Hospital, Shanxi Medical University, Taiyuan, 'Otolaryngology Head \& Neck Surgery Research Institute, Shanxi Medical University, Taiyuan, ${ }^{\mathrm{d}}$ The Key Scientific and Technological Innovation Platform for Precision Diagnosis and Treatment of Head and Neck Cancer, Shanxi Province, Taiyuan, e'Department of Otolaryngology Head \& Neck Surgery, The First Hospital, Jinzhou Medical University, Jinzhou, fDepartment of Pathology, Shanxi Cancer Hospital, Shanxi Medical University, Taiyuan, 9Department of MRI \& CT, Shanxi Cancer Hospital, Shanxi Medical University, Taiyuan, hModern Research Center for Traditional Chinese Medicine, Shanxi University, Taiyuan, China

\section{Key Words}

Laryngeal squamous cell carcinoma $\cdot \mathrm{CD} 133+\mathrm{CD} 44+\cdot$ Cancer stem cells $\bullet$ Whole transcriptome analysis • Circular RNA

\begin{abstract}
Background/Aims: CD133+CD44+ cancer stem cells previously isolated from laryngeal squamous cell carcinoma (LSCC) cell lines showed strong malignancy and tumorigenicity. However, the molecular mechanism underlying the enhanced malignancy remained unclear. Methods: Cell proliferation assay, spheroid-formation experiment, RNA sequencing (RNAseq), miRNA-seq, bioinformatic analysis, quantitative real-time PCR, migration assay, invasion assay, and luciferase reporter assay were used to identify differentially expressed mRNAs, IncRNAs, circRNAs and miRNAs, construct transcription regulatory network, and investigate functional roles and mechanism of circRNA in CD133+CD44+ laryngeal cancer stem cells. Results: Differentially expressed genes in TDP cells were mainly enriched in the biological processes of cell differentiation, regulation of autophagy, negative regulation of cell death, regulation of cell growth, response to hypoxia, telomere maintenance, cellular response to gamma radiation, and regulation of apoptotic signaling, which are closely related to the Y. Wu, Y. Zhang, M. Niu and Y. Shi contributed equally to this work.

Wei Gao, Professor

and Shuxin Wen, Professor

Shanxi Key Laboratory of Otorhinolaryngology Head and Neck Cancer, No. 85 Jiefang

Road, South, Taiyuan 030001, Shanxi (China)

Tel.86351-4867076, E-Mail gaoweisxent@sxent.org,wsxsx@sxent.org
\end{abstract}


malignant features of tumor cells. We constructed the regulatory network of differentially expressed circRNAs, miRNAs and mRNAs. qPCR findings for the expression of key genes in the network were consistent with the sequencing data. Moreover, our data revealed that circRNA hg19_circ_0005033 promotes proliferation, migration, invasion, and chemotherapy resistance of laryngeal cancer stem cells. Conclusions: This study provides potential biomarkers and targets for LSCC diagnosis and therapy, and provide important evidences for the heterogeneity of LSCC cells at the transcription level.

(C) 2018 The Author(s)

Published by S. Karger AG, Basel

\section{Introduction}

Laryngeal cancer is one of the most common malignant tumors worldwide; it affects approximately 10, 000 Americans per year [1]. Laryngeal squamous cell carcinoma (LSCC) is the main type of aggressive neoplasms of the larynx. Despite recent advances in surgical treatments such as functional laryngeal surgery and oncological treatment including radiotherapy and chemotherapy, the prognosis of advanced LSCC remains poor due to tumor recurrence or metastasis $[2,3]$. Therefore, understanding the mechanism underlying the invasion and metastasis of LSCC could lead to the development of novel treatment strategies.

Cancer stem cells are considered the origin of cancer cells, which are closely associated with therapeutic resistance and cancer recurrence [4, 5]. Our previous study showed that CD133+CD44+ cancer stem cells isolated from the LSCC cell lines Hep2 and TU-177 by magnetic-activated cell sorting (MACS) exhibited enhanced cell viability, migration and invasive capability, colony-formation ability, and resistance to chemotherapy and irradiation. Furthermore, in nude mice, tumorigenicity was higher for CD133+CD44+ LSCC stem cells than CD133+ or CD44+ LSCC stem cells [6]. Therefore, investigating the mechanisms underlying CD133+CD44+ LSCC cancer cells would help with treatment and prognosis of LSCC.

MicroRNA (miRNA) is a type of small ( $\sim 22 \mathrm{nt})$ single-stranded RNA that exerts biological functions by base-pairing to target genes (mRNA) to cause mRNA degradation [7]. Circular RNA (circRNA) is a new class of single-stranded RNA. CircRNAs regulate gene expression via specific miRNA binding sites, which function as a competing endogenous RNA (ceRNA) to bind miRNA, thus restoring expression of miRNA target $[8,9]$. Moreover, long non-coding RNA (lncRNA), which regulates gene expression at various levels, has emerged as a major source of biomarkers and targets for cancer therapeutics [10].

In this study, we used whole-transcriptome sequencing to analyze differentially expressed circRNAs, miRNAs, IncRNAs, and mRNAs in CD133+CD44+ LSCC stem cells. We also used integrated analysis to reveal the regulatory relation among the differentially expressed circRNAs, miRNAs and mRNAs, and verified the expression of key circRNAs, miRNAs and mRNAs in TDP and TDN cells by qPCR. Moreover, we investigated the role of core circRNA in regulation of proliferation, migration, invasion, and chemotherapy of cancer stem cells. Our results give new insights into the molecular mechanisms of cancer stem cells and suggest potential novel biomarkers for LSCC therapy.

\section{Materials and Methods}

Cells and culture

TU-177 LSCC cells were obtained from Bioleaf Biotech Corp. (Shanghai). The LSCC cell line Hep2 and HEK293T cell line were obtained from the China Center for Type Culture Collection (Wuhan, China). LSCC cell lines M2e, M4e, TU212 and TU686 were purchased from the Advanced Research Center of Central South University (Hunan, China). HEK293T and Hep2 cells were cultured in DMEM supplemented with $10 \%$ fetal bovine serum (Biological Industries, Cromwell, CT). TU-177 cells were cultured in MEM supplemented with $10 \%$ fetal bovine serum. M2e, M4e, TU212 and TU686 cells were maintained in RPMI1640 supplemented with $10 \%$ fetal bovine serum. All cells were maintained in an incubator at $37{ }^{\circ} \mathrm{C}, 5 \% \mathrm{CO} 2$ in a humidified atmosphere. 


\section{Cellular Physiology Cell Physiol Biochem 2018;47:1696-1710 \begin{tabular}{l|l} 
and Biochemistry Published online: June 27, 2018 & $\begin{array}{l}\text { (c) } 2018 \text { The Author(s). Published by S. Karger AG, Basel } \\
\text { www.karger.com/cpb }\end{array}$
\end{tabular} \\ Wu et al.: Transcriptomic Analysis of LSCC Stem Cells}

\section{Inhibition of cells with cisplatin}

IC50 values in response to cisplatin treatment for the above cells were determined by using an xCELLigence RTCA S16 instrument (ACEA Biosciences, Hangzhou, China) following the manufacturer's protocol. Briefly, one day before cisplatin treatment, $5 \times 10^{3}$ cells were seeded on 16 -well E-Plates and incubated at room temperature for $30 \mathrm{~min}$, then the E-Plate was transferred to an RTCA station in a CO2 incubator. The cell index was detected every $15 \mathrm{~min}$. When the cell index was 0.8 , various concentrations of cisplatin $(0,1,2.5,5,10,20,50,100 \mu \mathrm{g} / \mathrm{ml})$ were added to the wells, and the cell index was detected every $15 \mathrm{~min}$, up to $72 \mathrm{~h}$. IC50 values in response to cisplatin for each cell line were calculated according to the cell index changes after treatment.

\section{Cells sorting, RNA extraction, and transcriptome sequencing}

CD $133^{+}{ }^{+} D 44{ }^{+}$cells (hereafter TDP cells) and CD133-CD44- cells (hereafter TDN cells) were isolated from LSCC TU-177 cells, and spheroid-formation experiments were conducted as described [6]. Total RNA was extracted by using TRIzol reagent (Invitrogen, Carlsbad, CA) as the manufacturer instructed, followed by DNase I treatment to remove DNA contamination. Equal quantities of RNA from 3 samples in one group were mixed. The lncRNA library (including lncRNAs, mRNAs, and circRNAs) and small RNA library were prepared by Novogene Bioinformatics Technology (Beijing, China). Agilent 2100 Bioanalyzer was used to qualify and quantify the sample library. The library products were sequenced on Illumina HiSeq 2000/4000 platform by Novogene Bioinformatics Technology. The sequencing data were submitted to GEO (GSE106280, GSE106283).

\section{Processing of sequencing data}

Data processing of raw reads was quality checked by using fastqc v0.10.1 (http://www.bioinformatics. babraham.ac.uk/projects/fastqc/) and trimmed for low-quality bases and adaptors if necessary by using fastx_toolkit V0.0.13 (http://hannonlab.cshl.edu/fastx_toolkit/). The expression patterns of lncRNAs, circRNAs, mRNAs and miRNAs were analyzed by comparing TDP versus TPT and TDN versus TPT cells.

\section{Analysis of mRNA and IncRNA sequencing data}

Raw reads in the fastq format were first processed by in-house perl scripts. Clean reads were obtained by removing reads containing an adapter, reads containing ploy- $\mathrm{N}$, and low-quality reads from raw data. Clean reads were mapped to the reference genome by using HISAT2 v2.0.4 with "--rna-strandness RF" and other parameters set as a default. The mapped reads of each sample were assembled by using StringTie v1.3.1 [11] in a reference-based approach. The assembled transcripts were analyzed for coding potential by using Coding-Non-Coding-Index (CNCI), Coding Potential Calculator (CPC), Pfam-sca, and phylogenetic codon substitution frequency (PhyloCSF) algorithms. Transcripts without coding potential were the candidate set of IncRNAs. Fragments per kilobase of exon per million reads (FPKMs) for both lncRNAs and coding genes in each sample were calculated by using Cuffdiff v2.1.1 [12]. Gene FPKMs were computed by summing the FPKMs for transcripts in each gene group, then the differentially expressed mRNAs and lncRNAs were calculated by using the FPKM values for each gene.

\section{Analysis of miRNA sequencing data}

The clean reads were mapped to the hg19 reference genome by using Bowtie v1.1.2 to identify small RNA loci including miRNAs. The miRNAs in each group were identified based on the known miRNAs downloaded from the miRBase database v21.0 [13]. The expression of miRNAs in each sample was normalized by using transcripts per million (TPM), and read counts of annotated sequences were normalized by the trimmed mean of M values (TMM). Differentially expressed miRNAs were then identified by using the DEGseq R package [14] with $\mathrm{p}<0.01$ and $\log 2 \mathrm{FC}$ (fold change) $\mid>1$. MiRanda, a widely used open-source software for target prediction, was used to identify the most probable miRNA targets. Finally, GO and KEGG pathway analysis of miRNA target genes involved use of R clusterProfiler (http://bioconductor.org/packages/2.8/ bioc/html/clusterProfiler.html).

\section{Analysis of circRNA sequencing data}

CircRNAs were identified by using the find_circ software, followed by matching with the circRNA data from the circBase database [15]. The expression of known and predicted circRNAs was normalized by 


\begin{tabular}{|c|c|c|}
\hline Cellular Physiology & Cell Physiol Biochem 2018;4 & 7:1696-1710 \\
\hline Biochemistrv & $\begin{array}{l}\text { Dor: 10.1159/000490992 } \\
\text { Published online: }\end{array}$ & $\begin{array}{l}\text { O } 2018 \text { The Author(s). Published by S. Karger AG, Basel } \\
\text { www.karger.com/cpb }\end{array}$ \\
\hline
\end{tabular}

TPM, and the differential expression analysis involved use of DEGseq. CircRNAs with $\mathrm{q}<0.01$ and $\mid \log 2 \mathrm{FC}$ (fold change) $\mid>1$ were considered differentially expressed. Because circRNAs are mainly derived from the exons of protein-coding genes, GO terms and KEGG pathways enriched by the downstream protein-coding genes of circRNAs were identified by the hyper-geometric distribution. In addition, circRNA-miRNA interactions were predicted by using the miRanda prediction algorithm.

\section{GO and KEGG enrichment analysis}

To understand the potential roles of differentially expressed genes, GO and KEGG enrichment analyses were used with the online program DAVID (https:// david.ncifcrf.gov/home.jsp) [16].

Co-regulatory network construction based on differentially expressed mRNAs, miRNAs and circRNAs

CeRNA-based analysis of circRNAs, miRNAs and mRNAs involved use of miRNA binding-site prediction of circRNAs, miRNA target gene prediction, and correlation analysis. The regulatory network was constructed by using the bioinformatics package Cytoscape [17], widely used for biological network visualization and data integration.

Real-time quantitative PCR ( $P P C R$ ) validation of circRNAs, mRNAs and miRNAs

Differentially expressed circRNAs, mRNAs, miRNAs of $p$ value less than 0.05 and in the circRNAmiRNA-mRNA network, and the highest expressed lncRNAs were validated by qPCR. Briefly, total RNA was isolated from TDP, TDN and TPT cells by using TRIzol reagent (Invitrogen). For circRNAs and mRNAs, firststrand cDNA was synthesized by using the HiScript II 1st Strand cDNA Synthesis Kit (R211-02, Vazyme, Nanjing, China). For miRNAs, first-strand cDNA was synthesized by using an All-in-One miRNA first-strand cDNA Synthesis Kit (GeneCopoeia, Inc., Rockville, MD, USA). qPCR involved use of the ChamQ SYBR qPCR Master Mix (Q331-02, Vazyme) on an ABI 7500 FAST real-time PCR system (Applied Biosystems, Foster City, CA, USA). The qPCR conditions were $95^{\circ} \mathrm{C}$ for $30 \mathrm{sec}$, followed by 40 cycles of $95^{\circ} \mathrm{C}$ for $10 \mathrm{sec}$ and $60^{\circ} \mathrm{C}$ for $30 \mathrm{sec}$. The specificity of the primer amplicons was examined by melting curve analysis. The comparative Ct method was used for quantifying target circRNA, mRNA and miRNA expression normalized to that of $18 \mathrm{~S}$ rRNA or RNU6 (the internal control). qPCR primers used in this study were list in Table 1.

\section{Transfection of RNA oligos}

Knockdown of hg_circ_0005033 was performed using siRNAs targeting hg_circ_0005033, the siRNA sequences were as follows, si-circ_0005033-1: sense: GAAUGGAUUGUGGAAAGAA, antisense: UUCUUUCCACAAUCCAUUC; si-circ_0005033-2: sense: GGAUUGUGGAAAGAAGCUC, antisense: GAGCUUCUUUCCACAAUCC; si-NC: sense: AUGCGAGAUAUGCGUAAGG, antisense: CCUUACGCAUAUCUCGCAU. TDP cells were transfected with siRNAs, miRNA mimics, and miRNA inhibitor using lipofectamine 3000 transfection reagent (Thermo Fisher Scientific, Waltham, MA) for $48 \mathrm{~h}$, and then knockdown efficiency and related genes expression were detected.

Cell proliferation and chemotherapy resistance assay

Cell proliferation was tested using Cell count kit-8 (CCK-8, Dojindo, Japan). Briefly, $1 \times 10^{3}$ TDP cells were seeded into 96-well plates, and $10 \mu \mathrm{l}$ CCK-8 solution was added to each well at the indicated time 


\section{Cellular Physiology Cell Physiol Biochem 2018:47:1696-1710 and Biochemistry Published online: June 27, $2018 \quad \begin{aligned} & \text { DOI: 10.1159/000490992 } 2018 \text { The Author(s). Published by S. Karger AG, Basel } \\ & \text { www.karger.com/cpb }\end{aligned}$ \\ Wu et al.: Transcriptomic Analysis of LSCC Stem Cells}

points. The cells were incubated at $37^{\circ} \mathrm{C}$ for 2 hours, and the absorbance at $450 \mathrm{~nm}$ was measured using Multiskan FC Microplate Photometer (Thermo Fisher Scientific). Chemotherapy resistance assay was performed by treating cells with various concentrations of cisplatin and detected using Cell count kit-8. The data are representative of three individual experiments.

\section{Migration and invasion assay}

Transwell chamber assay was performed for migration and invasion analysis of cells as described previously [6]. Briefly, cells were resuspended with culture medium containing 1\% FBS (Biological Industries, Cromwell, CT). $200 \mu \mathrm{L}$ cell suspension $(1 \times 105$ cells) was added into the Transwell chamber (BD Biosciences, Franklin Lakes, NJ). The lower chamber was added $500 \mu \mathrm{L}$ medium containing 30\% FBS for 48 h. After $48 \mathrm{~h}$ of incubating at $37^{\circ} \mathrm{C}$ with $5 \% \mathrm{CO} 2$, cells were rinsed with PBS and fixed in $0.5 \%$ methanol for $30 \mathrm{~min}$, stained with $0.1 \%$ crystal violet for $20 \mathrm{~min}$, and rinsed with PBS. The un-invaded cells were wiped with a cotton swab, and cells penetrating the membrane were observed by microscopy and counted. Each experiment was performed in triplicate.

\section{Luciferase reporter assay}

The wild type (WT) cDNA fragments of hg_circ_0005033 containing predicted miR-4521 binding sites were amplified and mutated fragments (Mutant) were generated through overlap extension PCR, then WT and mutant fragments were cloned into the psiCHECK-2 (Promega, Madison, WI), respectively. For luciferase reporter assay, HEK293T cells were co-transfected with wild type vector or mutant vector and Hsa-miR-4521 mimics or control mimics using Lipofectamine 3000 (Thermo Fisher Scientific). After $48 \mathrm{~h}$ of transfection, the luciferase activity was detected using dual-luciferase reporter assay kit (Promega) and normalized to Renilla luciferase activity. Each experiment was performed in triplicate.

\section{Statistical analysis}

Independent two-sample $t$ test was used for qPCR analysis. $\mathrm{P}<0.05$ was considered statistically significant. Statistical analysis involved use of SPSS 13.0.

\section{Results}

Identification of differentially expressed IncRNAs, mRNAs, miRNAs and circRNAs in CD133+CD44+ cancer stem cells of LSCC

Recently, we isolated CD133+CD44+ cells from the LSCC cell lines Hep2 and TU-177 by MACS. Functional analysis demonstrated that these cells had stronger malignant behavior than parental cells and CD133+ or CD44+ cells, including proliferation, migration and invasion, resistance to chemotherapeutic drugs and radiation, and in vivo tumorigenicity. Importantly, the high expression of stem-cell markers SOX2 and OCT4 indicated that these CD133+CD44+ cells were LSCC stem cells [6]. To determine whether CD133+CD44+ LSCC stem cells contribute to the resistance of LSCC cells to chemotherapy, we compared the ratio of the CD133+CD44+ subpopulation and IC50 values after cisplatin treatment in six LSCC cell lines (Hep2, TU-177, TU686, TU212, M2e, and M4e). Hep2 cells had the highest ratio of CD133+CD44+ subpopulation, followed by TU-177, M2e, M4e, TU686, then TU212 cells with the lowest ratio of CD133+CD44+ subpopulation (Fig. 1A). The ratio of CD44+ subpopulation varied greatly among these 6 LSCC cell types. TU-177 cells had the highest ratio, followed by M2e, Hep2, M4e, TU686, and TU212 (Fig. 1A). As expected, Hep2 cells exhibited the highest IC50 value with cisplatin treatment, and TU212 cells had the lowest value (Fig. 1B). Moreover, TU-177 cells showed the second highest IC50 value with cisplatin treatment, followed by M2e, M4e, and TU686 cells (Fig. 1B). These results, together with our previous data [6], indicate that CD133+CD44+ LSCC stem cells are the main contributors to cisplatin resistance of LSCC cells, and the CD44+ subpopulation also contributed to cisplatin resistance.

The LSCC cell line Hep2 is known to be contaminated by Hela cells [18]. Since the ratio of CD133+CD44+ cells was found higher in TU-177 cells than M2e, M4e, TU686, and 
Fig. 1. Isolation, characterization and transcriptomic analysis of CD133+CD44+ LSCC stem cells. (A) Proportion of CD133+CD44+ cells in 6 LSCC cell lines. Ratio of CD133+CD44+ cells in LSCC cell lines Hep2, TU-177, M2e, M4e, TU686 and TU212 was measured by flow cytometry. (B) Hep2, TU177, M2e, M4e, TU686 and TU212 cells were treated with various concentrations of cisplatin, and the $\mathrm{IC}_{50}$ values of cells to cisplatin were determined by RTCA at 24, 48 and 72 h. Statistical difference calculated $\mathrm{p}$ values were shown above the bar. (C) Proportion of CD133+CD44+ cells in TU-177 cells before and after CD133 and CD44 antibodies magnetic beads treatment. (D) Sphere formation of TDP, TPT, and TDN cells. (E-H) Number of differentially expressed (E) IncRNAs, (F) Protein-coding genes (mRNAs), (G) miRNAs, and (H) circRNAs in TDP and TDN
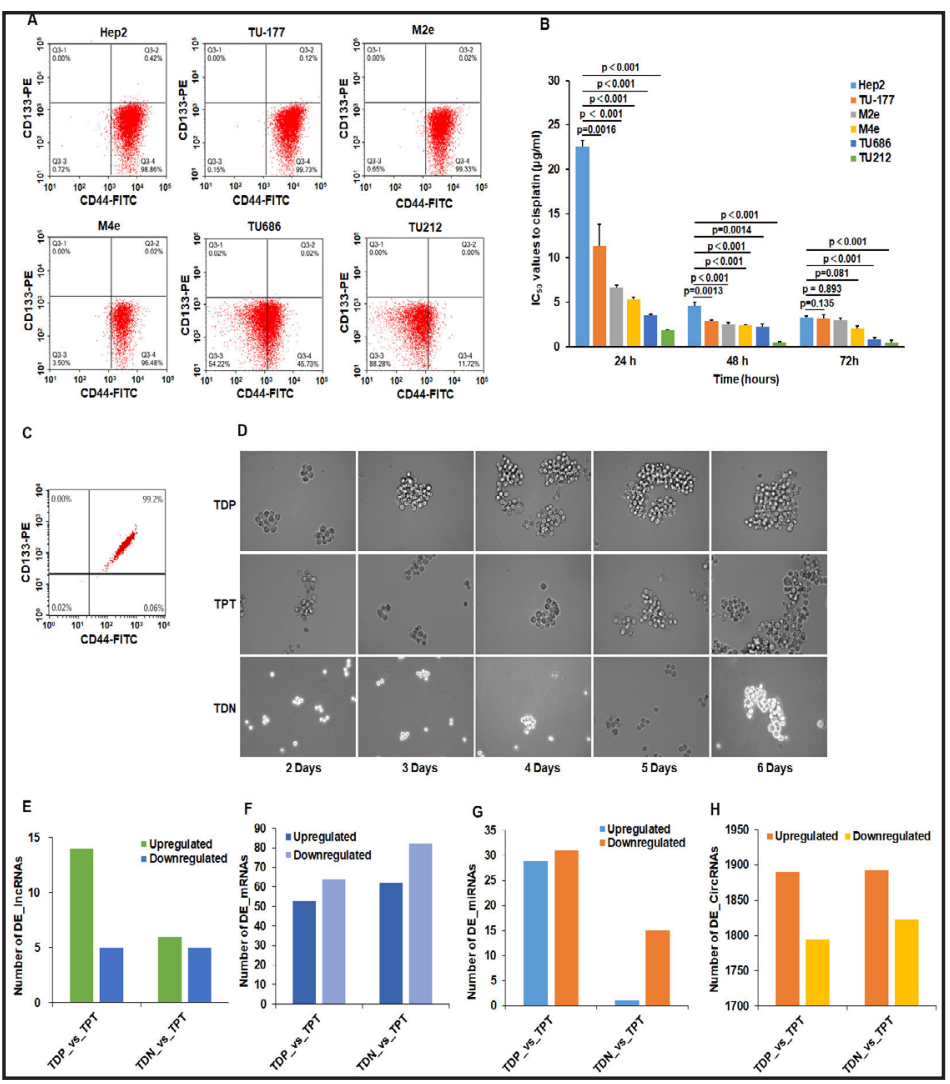
cells. Data are number of IncRNAs, mRNAs, miRNAs and circRNAs with fold change $>2$ and p<0.01. TDP: CD133+CD44+ cells; TDN: CD133CD44- cells; TPT: parental TU-177 cells.

TU212 cells, previously we isolated CD133+CD44+ LSCC stem cells from TU-177 cells and investigated their phenotypes [6]. In this study, we used TU-177 cells as a model to analyze the differential gene network of LSCC stem cells. The purity of CD133+CD44+ TU-177 cells isolated by MACS was evaluated by flow cytometry (Fig. 1C). Spheroid-formation experiments further confirmed the stem cell-like features of CD133+CD44+ TU-177 cells (Fig. 1D). Then, total RNA samples of CD133+CD44+, CD133-CD44-, and parental TU-177 cells (TPT) were subjected to RNA-sequencing (lncRNA, mRNA and circRNA) and miRNA-sequencing. After quality control, mapping and quantification of the sequencing data, differentially expressed lncRNAs, mRNAs, miRNAs and circRNAs were screened by fold change in expression and $p$ value (fold change $>2$, p value $<0.01$ ). Compared with the parental TU-177 cells, TDP cells showed differential expression of 19 lncRNAs, 117 mRNAs, 60 miRNAs and 3684 circRNAs (Fig. 1E-H, (for all online suppl. material, see www.karger.com/doi/ 10.1159/000490992, suppl. Table S1-8). In contrast, TDN cells showed differential expression of 11 lncRNAs, 144 mRNAs, 16 miRNAs and 3715 circRNAs (Fig. 1E-H, see online suppl. material, suppl. Table S1-8).

Functional analysis of differentially expressed mRNAs between TDP and TDN cells

With GO analysis for functional annotation, mRNAs upregulated in TDP cells were mainly enriched in biological processes of cell differentiation, regulation of autophagy, positive regulation of translation, negative regulation of cell death, reactive oxygen species metabolic process, regulation of cell growth, and cellular response to calcium ion (Fig. 2A). mRNAs downregulated in TDP cells were involved in caveolin-mediated endocytosis, negative regulation of protein tyrosine kinase activity, SMAD protein import into nucleus, response to hypoxia, telomere maintenance, cellular response to gamma radiation (Fig. 2B). 
mRNAs upregulated in TDN cells were significantly enriched in biological processes of skeletal muscle cell differentiation, regulation of cell growth, cellular response to corticotropin-releasing hormone stimulus, cell differentiation, DNA repair, intracellular receptor signaling pathway, positive regulation of nuclear-transcribed mRNA poly(A) tail shortening, negative regulation of apoptotic process, telomere maintenance, cellular response to extracellular stimulus, and cellular response to gamma radiation (Fig. 2C). In contrast, mRNAs downregulated in TDN cells were significantly enriched in caveolinmediated endocytosis, caveola assembly, cellular response to indole-3-methanol, positive regulation of I-kappaB kinase/NF-kappaB signaling, response to progesterone, regulation of cytokine production, negative regulation of epithelial cell differentiation, and cell division (Fig. 2D). Moreover, mRNAs downregulated in TDN cells were significantly involved in pathways of adherens junction, leukocyte transend othelial migration, cancer, focal adhesion, and regulation of actin cytoskeleton (Fig. 2D).

Functional analysis of differentially expressed miRNAs between TDP and TDN cells

To understand the potential role of differentially expressed miRNAs in TDP and TDN cells, we predicted possible target genes of these miRNAs, then annotated the functions of miRNA target genes by GO and KEGG pathway analysis. miRNAs upregulated in TDP cells were involved in heart development, tube morphogenesis, regulation of cell morphogenesis, positive regulation of neuron differentiation, as well as regulation of autophagy (see online

Fig. 2. Functional annotation of differentially expressed mRNAs in TDP and TDN cells. GO and KEGG pathway analysis of $(A, B)$ mRNAs upregulated and downregulated, respectively, in TDP cells and (C, D) mRNAs upregulated and downregulated, respectively, in TDN cells. TDP: CD133+CD44+ cells; TDN: CD133-CD44- cells; MF: molecular function; CC: cellular component; BP: biological process. Number above the bar graph indicates number of genes enriched in the specific biological process/signaling pathway.

Fig. 3. Comparison of potential functions of differentially expressed miRNAs in TDP and TDN cells. Common and specific biological processes of (A) miRNAs upregulated and downregulated in TDP cells and (B) miRNAs downregulated in TDP and TDN cells. (C) TDP up/down. microRNA: miRNAs upregulated or downregulated in CD133+CD44+ cells; TDN up/down. microRNA: miRNAs upregulated or downregulated in CD133-CD44cells.
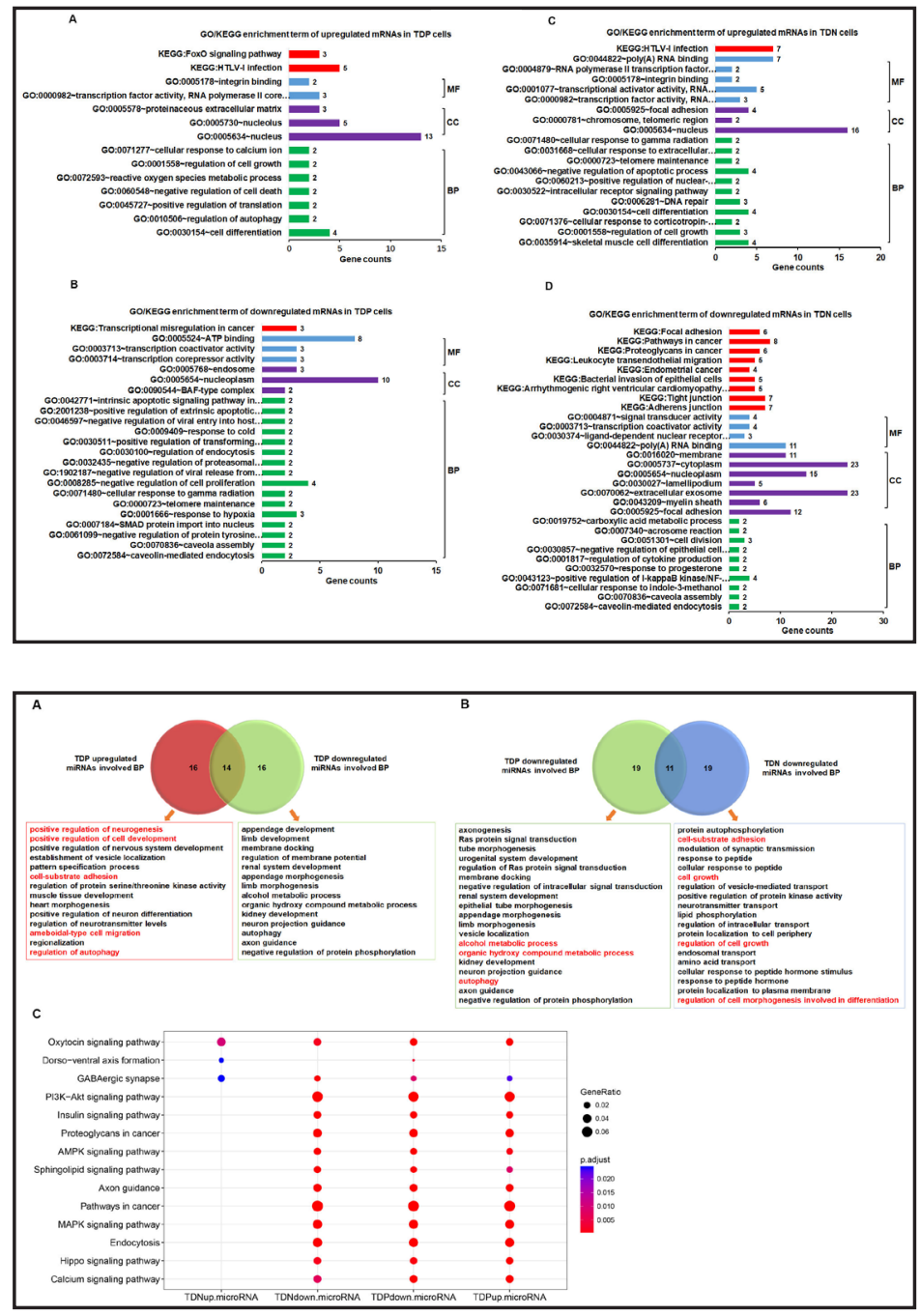
suppl. material, Suppl Table S9). In contrast, miRNAs downregulated in TDP cells were mainly enriched in small GTPase-mediated signal transduction, axonogenesis, axon development, alcohol metabolic process, and cell-matrix adhesion (see online suppl. material, Suppl. Table S10). Interestingly, as compared with miRNAs downregulated in TDP cells, those upregulated in TDP cells were specifically enriched in biological processes of positive regulation of neurogenesis, positive regulation of cell development, cell-substrate adhesion, ameboidaltype cell migration, and regulation of autophagy (Fig. 3A). Moreover, as compared with miRNAs downregulated in TDN cells, those downregulated in TDP cells were specifically involved in processes of alcohol metabolic process, organic hydroxy compound metabolic process, and autophagy, whereas miRNAs downregulated in TDN cells were mainly enriched in biological processes of cell-matrix adhesion, cell growth, regulation of cell growth, as well as regulation of cell morphogenesis involved in differentiation (Fig. 3B).

Pathway analysis showed that the upregulated miRNA targets of TDN cells were mainly enriched in the oxytocin signaling pathway, whereas downregulated miRNA targets of TDN cells and down- and upregulated miRNA targets of TDP cells were mainly enriched in PI3KAkt signaling pathway, pathways in cancer, and endocytosis (Fig. 3C).

\section{Differentially expressed circRNAs in TDP and TDN cells mainly involved in metabolic} processes

Integrated analysis of the differentially expressed miRNAs, mRNAs and circRNAs predicted circRNA-targeted mRNAs. We further investigated the possible role of circRNAtargeted mRNAs by GO and KEGG analysis. Downstream protein-coding genes of differentially expressed circRNAs in TDP and TDN cells were enriched in various metabolic and catabolic processes, such as cellular macromolecule metabolic, nucleic acid metabolic, primary metabolic, cellular nitrogen-compound metabolic, cellular protein catabolic, and cellular macromolecule catabolic processes (Fig. 4A, B, see online suppl. material, Suppl. Table S11, S12).

Pathway analysis revealed that differentially expressed circRNAs in TDP cells were mainly involved in ubiquitin-mediated proteolysis, RNA transport, lysine degradation, and the cell cycle (Fig. 4C, see online suppl. material, Suppl. Table S13). In contrast, differentially expressed circRNAs in TDN cells were significantly enriched in pathways of ubiquitinmediated proteolysis, RNA transport, cell cycle, colorectal cancer, and adherens junction (Fig. 4D, see online suppl. material, Suppl. Table S14).

Fig. 4. Functional annotation of predicted targets of differentially expressed circRNAs. The top 20 biological processes enriched for differentially expressed circRNAs of (A) TDP cells and (B) TDN cells plotted according to $\mathrm{p}$ values. The top 20 KEGG pathways enriched for downstream protein-coding genes of differentially expressed circRNAs in (C) TDP cells and (D) TDN cells.

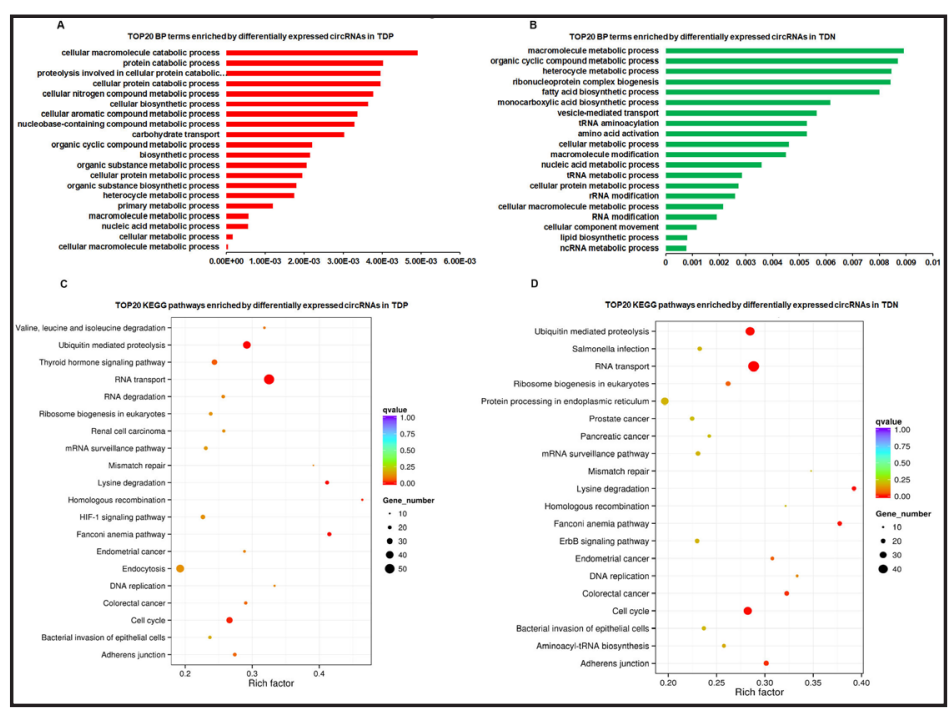


mRNA-miRNA-circRNA regulatory network and functional enrichment of key genes

We investigated miRNA-mRNA interaction pairs with an opposite expression trend (e.g., the miRNA was upregulated and the mRNA was downregulated) and obtained 458 and 91 miRNA-mRNA interaction pairs by comparing TDP versus TPT cells and TDN versus TPT cells, respectively. We also analyzed miRNA-circRNA interaction pair with opposite expression trend and obtained 439 and 126 miRNA-circRNA interaction pairs in TDP versus TPT cells and TDN versus TPT cells, respectively.

The regulatory network of TDP includes 731 nodes and 897 edges. The mRNAs including PEAK1, NFAT5, SMAD2, KCNQ5, PDLIM5, REV3L, UHRF1BP1 and CTBP2 had a higher degree than the others (Fig. 5A). Functional analysis showed that mRNAs in the TDP ceRNA network were enriched in biological processes of cell-cell adhesion, cell migration, double-strand break repair, and pathways in cancer (Fig. 5B). Because of the large size of the network, we selected the mRNA-miRNA-circRNA connection pairs that were regulated by two or more miRNA and constructed the core regulatory network. The core network of TDP contains three, four and six upregulated mRNAs, miRNAs and circRNAs, respectively; and four, five, and four downregulated mRNAs, miRNAs and circRNAs, respectively (Fig. 5C).

The TDN ceRNA network was much larger than the TDP network, and miR-4521, miR1-3p, miR-133a-3p, miR-143-3p, miR-206 and miR-451a were the crucial miRNAs in this network (Fig. 5D). mRNAs in the TDN ceRNA network were significantly enriched in the IRE1-mediated unfolded protein response, cell-cell adhesion, cell migration, and cell division. Some hub node genes were related to pathways of protein processing in endoplasmic reticulum, such as SSR1 and INTS6. However, hub node genes PEAK1, WWC1, NFATC2 and ABL2 were enriched in the process of cell migration (Fig. 5E).

\section{qPCR validation of the differentially expressed genes}

To better understand the expression levels of the key differentially expressed genes in TDP and TDN cells, we used qPCR to determine mRNA, circRNA and miRNA levels in the core ceRNA regulatory network. The expression of the circRNAs hg19_circ_0005033, hg19_ circ_0004481, and hg19_circ_0003610; mRNAs PDLIM5, STAT5A, LGI4, VANGL1, SMAD2, and DCTN4; and miRNAs miR-328-3p, miR-4521, miR-451a, and miR-4661-5p was consistent with sequencing data in TDP cells (Fig. 6A-C).

Fig. 5. Integrated analysis of differentially expressed circRNAs, miRNAs and mRNAs. (A) The mRNA-miRNA-circRNA regulatory network of TDP cells. (B) GO and KEGG analysis of mRNAs in the mRNA-miRNA-circRNA regulatory network of TDP cells. (C) The core regulatory network extracted from the overall mRNA-miRNA-circRNA regulatory network of TDP cells. (D) The mRNA-miRNA-circRNA regulatory network of TDN cells. For A, C and D, green and red represent down- and upregulation, respectively. Triangular, arrow and round indicate microRNAs, circRNAs and mRNAs, respectively. (E) GO analysis of mRNAs in the mRNA-miRNA-circRNA regulatory

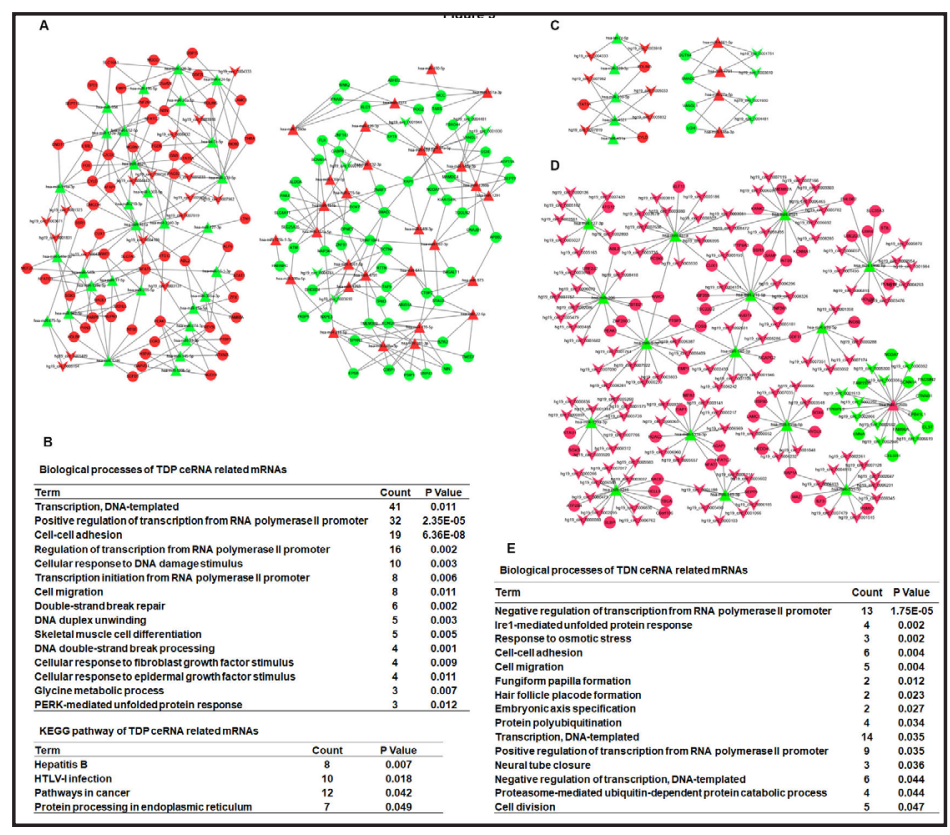
network of TDP cells. 
Fig. 6. Validation of the expression of mRNAs, circRNAs and miRNAs in TDP and TDN cells. TDP and TDN cells were isolated from TU177 cells by magnetic-activated cell sorting, then total RNA was purified. qPCR analysis of expression of circRNAs (A, D), mRNAs (B, E), and miRNAs (C, F). (G) Expression of IncRNA RP1179P5.2 and RP11-1100L3.8 in TDP or TDN cells was determined by

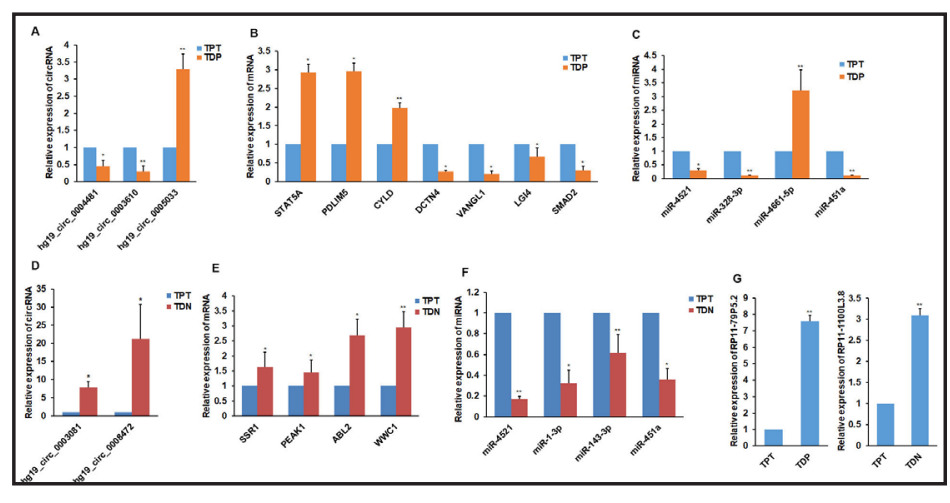
qPCR. Data were represented as mean \pm SD of three independent experiments. ${ }^{*} \mathrm{p}<0.05,{ }^{* *} \mathrm{p}<0.01$. TDP: CD133+CD44+ cells. TDN: CD133CD44- cells. TPT: parental TU-177 cells.

Fig. 7. Knockdown of hg19_circ_0005033 inhibits proliferation, migration, and invasion of LSCC stem cells. (A) Knockdown efficiency of hg circ_0005033 was determined by qPCR. (B) Proliferation ability of hg_circ_0005033 knockdown TDP cells and control cells was detected by CCK8 assay. (C) Transwell assay of migration and invasion ability of hg_circ_0005033 knockdown TDP cells. (D) hg_circ_0005033 knockdown TDP cells and control cells were treated with cisplatin at the indicated concentration for $48 \mathrm{~h}$, then cell viability was determined by CCK-8 assay. (E) Putative miR-4521 biding sites on hg_circ_0005033 predicted using seedVicious v1.1. (F) Luciferase

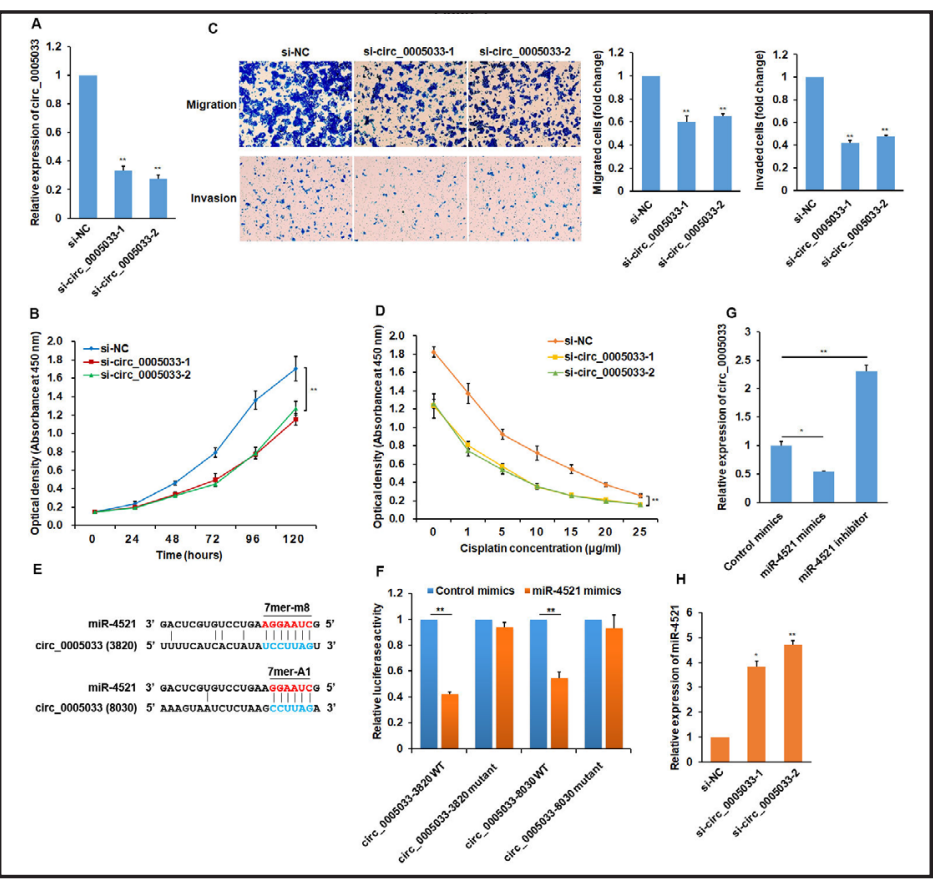
reporter assay validated the interaction between hg_circ_0005033 and miR-4521. (G) Expression level of hg_circ_0005033 in TDP cells transfected with miR-4521 mimics or inhibitor. Expression level of hg_circ_0005033 was determined by qPCR. (H) Expression level of miR-4521 in TDP cells transfected with siRNAs targeting hg_circ_0005033. Data were represented as mean \pm SD of three independent experiments. ${ }^{*} \mathrm{p}<0.05,{ }^{* *} \mathrm{p}<0.01$.

qPCR revealed upregulation of hg19_circ_0003081 and hg19_circ_0008472 as well as SSR1, PEAK1, ABL2 and WWC1 and downregulation of miR-4521, miR-1-3p, miR-143-3p and miR-451a in TDN cells, which agreed with the sequencing data (Fig. 6D-F). Moreover, we investigated the expression of IncRNAs in TDP and TDN cells by qPCR. The lncRNA RP1179P5.2 was upregulated in TDP cells, and RP11-1100L3.8 was upregulated in TDN cells (Fig. $6 \mathrm{G})$. Taken together, these data confirm that TDP and TDN cells exhibit a circRNA-miRNAmRNA transcription regulatory network, which represents the foundation of the phenotype of LSCC stem cells. 


\section{Cellular Physiology Cell Physiol Biochem 2018;47:1696-1710 and Biochemistry Published online: June 27, $2018 \quad \begin{aligned} & \text { DOI: 10.1159/000490992 } 2018 \text { The Author(s). Published by S. Karger AG, Basel } \\ & \text { www.karger.com/cpb }\end{aligned}$ \\ Wu et al.: Transcriptomic Analysis of LSCC Stem Cells}

Knockdown of hg19_circ_0005033 inhibited TDP cells proliferation, migration, invasion, and cisplatin resistance

CircRNA plays critical roles in cancer carcinogenesis. hg19_circ_0005033 was one of the upregulated circRNAs in TDP cells. We explored the function of hg19_circ_0005033 in TDP cells through loss-of-function experiments. siRNAs transfection significantly knockdown the expression of hg19_circ_0005033 in TDP cells (Fig. 7A). Knockdown of hg19_circ_0005033 significantly inhibited proliferation of TDP cells (Fig. 7B). Moreover, transwell assay exhibits that knockdown of hg19_circ_0005033 inhibited migration and invasion ability of TDP cells (Fig. 7C). We further investigated the role of hg19_circ_0005033 in regulation of chemotherapy resistance of TDP cells. Results showed that hg19_circ_0005033 knockdown enhanced the sensitivity of TDP cells to cisplatin (Fig. 7D). These results indicated that the circRNA hg19_circ_0005033 plays important roles in proliferation, migration, invasion, and chemotherapy resistance of TDP cells.

circRNA functions as miRNA sponge was reported [19]. In the present study, the potential miRNA binding sites of hg19_circ_0005033 was predicted using seedVicious v1.1 (http:// seedvicious.essex.ac.uk/) (Fig. 7E). Luciferase reporter assay demonstrated that miR4521 binds to hg19_circ_0005033, resulting in reduction of luciferase activity of wild-type reporter constructs (Fig. 7F). Moreover, overexpression of miR-4521 downregulated hg19. circ_0005033 in TDP cells, while inhibition of miR-4521 upregulated hg19_circ_0005033 (Fig. 7G). Furthermore, Knockdown of hg19_circ_0005033 significantly upregulated miR4521 (Fig. 7H). Collectively, these data revealed that hg19_circ_0005033 functions as ceRNA to upregulate miR-4521 targeted mRNAs.

\section{Discussion}

Cancer stem cells are the driving force of tumor growth, migration, metastasis and therapeutic resistance. Therefore, investigating the features and underlying mechanisms of cancer stem cells hold great promise for cancer diagnosis and therapy [20]. Stem cells in head and neck cancer, especially laryngeal cancer, have been isolated and characterized $[21,22]$. Previously, we isolated CD133+CD144+ cancer stem cells from LSCC cell lines, and functional study demonstrated stronger malignant behaviors in CD133+CD144+ LSCC stem cells than CD133+ or CD44+ LSCC stem cells, CD133-CD44- LSCC cells, and parental cells [6]. However, the mechanism underlying LSCC stem cells remained unclear. In this study, we comprehensively analyzed the differential expression profiles of IncRNAs, mRNAs, circRNAs and miRNAs in TDP and TDN cells by whole-transcriptome sequencing. The data revealed that TDP and TDN cells have a unique and complex transcription regulatory network as compared with the parental TPT cells. Differentially expressed genes explained the differences in phenotype between TDP and TDN cells. Functional analysis revealed that differentially expressed mRNAs and miRNAs in TDP cells were involved in biological processes of autophagy, cell growth, cell differentiation, telomere maintenance, and response to hypoxia. Autophagy plays crucial roles in cancer, recent study revealed that enhanced autophagic activity was detected in ovarian cancer stem cells, and inhibition of autophagy reduced chemoresistance and self-renewal [23]. Activated telomere maintenance mechanism allows prolonged survival of the cells, which considered as a hallmark of cancer cells [24]. Therefore, differentially expressed genes that enriched in these biological processes and pathways might be important contributors of the enhanced malignancy of LSCC stem cells.

Integrated analysis is an important approach to understanding the regulatory relationships of large-scale transcriptomic sequencing data $[25,26]$. In the present study, we used integrated analysis of circRNAs, miRNAs, and mRNAs to construct TDP and TDN specific transcription regulatory networks. Furthermore, we validated the expression of genes at the core nodes of the circRNA-miRNA-mRNA regulatory network by qPCR. PDLIM5, which was upregulated in TDP cells, functions as an oncogene in cancer by promoting cell proliferation and migration $[27,28]$. Moreover, miR-328, which interacts with PDLIM5, has 


\section{Cellular Physiology Cell Physiol Biochem 2018;47:1696-1710 \\ \begin{tabular}{l|l} 
DOI: 10.1159/000490992 & $\begin{array}{l}\text { O 2018 The Author(s). Published by S. Karger AG, Basel } \\
\text { www.karger.com/cpb }\end{array}$
\end{tabular} \\ Wu et al.: Transcriptomic Analysis of LSCC Stem Cells}

been found downregulated in multiple cancers. miR-328 inhibits the survival of cancer cells and enhances the sensitivity of cancer to radiotherapy $[29,30]$. Furthermore, overexpression of miR-328 reversed drug resistance and inhibited cell invasion of cancer stem cell-like SP cells in colorectal cancer [31]. These data indicate that the circRNA-miRNA interaction upregulates the downstream target PDLIM5, thus promoting the malignancy of TDP cells.

PEAK1, ABL2 and WWC1 were previously found associated with cell migration. In migrating cells, PEAK1 is associated with the actin cytoskeleton and focal adhesion and involved in cancer progression [32]. The non-receptor tyrosine-protein kinase ABL2 plays critical roles in inhibiting the proliferation, migration and invasion of cancer cells [33, 34]. WWC1, which could restrict cell proliferation and increase cell apoptosis, is considered an important tumor suppressor gene $[35,36]$. Both ABL2 and WWC1 are potential targets of miR-451a. High expression of these tumor suppressors in TDN cells is consistent with the cells' lower ability for proliferation, migration, and invasion.

The pathway of protein processing in endoplasmic reticulum was enriched by mRNAs of both TDN and TDP cells, which points to a potential link between cancer and endoplasmic reticulum function. Previous studies have suggested the endoplasmic reticulum stress could determine apoptosis and cell death in cancers [37, 38]. SSR1, regulated by miR-4521, was associated with the pathway of protein processing. SSR1/Trap $\alpha$, which may have a general role in protein translocation [39], was found involved in mammalian heart development [40]. Moreover, miR-4521 was found involved in cancer pathogenesis [41, 42].

In addition, we noted that TDP ceRNA-related mRNAs were significantly enriched in the pathways hepatitis B and human T-lymphotropic virus infection and pathways in cancer. Infections causing human cancers has been widely proposed, and an estimated $12 \%$ of the worldwide incidence of human cancers was found attributed to viral infection [43]. Previous study indicated that human papillomavirus infection may play a role in the etiology of LSCC [44]. Our data revealed STAT5A as a target of miR-4521 and miR-339-5p, both of which also interact with hg19_circ_0005033. Particularly, STAT5A induces stem-like cell properties and the epithelial-to-mesenchymal transition in prostate cancer [45]. hg19_circ_0005033 may upregulate STAT5A to promote the phenotype of LSCC stem cells by binding miRNAs targeting STAT5A. Moreover, our data revealed that hg19_circ_0005033 targets miR-4521 and promotes proliferation, migration, invasion, and chemotherapy resistance in TDP cells. Previous study reported that miR-4521 was significantly decreased in sunitinib-resistant renal cell carcinoma [42]. Therefore, we speculated that circRNAs upregulated in TDP cells competed tumor-suppressive miRNAs, upregulating oncogenic mRNAs expression, thus maintaining malignant features of LSCC stem cells.

In conclusion, in this study, we investigated the transcription patterns of IncRNAs, mRNAs, circRNAs, and miRNAs of CD133+CD44+ LSCC stem cells by whole-transcriptome sequencing and integrated analysis. We reveal a unique circRNA-miRNA-mRNA regulatory network the supports the stem cell characteristics of CD133+CD44+ LSCC stem cells. Moreover, we investigated the role of core circRNA in regulation of phenotypes of LSCC stem cells. These findings provide potential biomarkers and targets for LSCC therapy, and provide pivotal evidence for the heterogeneity of LSCC cells at the transcription level.

\section{Acknowledgements}

We thank Jue Wang for the preparation of RNA samples, Huina Guo for qPCR experiments. S.W., and W.G. conceived and designed the experiments; Y.W., Y.Z., M.N., HL.L., Y.S., D.Y, F.L., Y.L., Y.B., R.Z., Z.L., HJ.L., and J.C. performed the experiments; Y.W., C.X., and J.S. analyzed the data; Y.W., W.G., and S.W. wrote the manuscript. All authors reviewed and agreed to submit this manuscript.

This work was supported by the National Natural Science Foundation of China (grant No. 81572670, 81402256, 81602394), China Postdoctoral Science Foundation (2016M591412, 2017M610174), Natural Science Foundation of Shanxi Province (2014011039-5, 


\section{Cellular Physiology Cell Physiol Biochem 2018;47:1696-1710

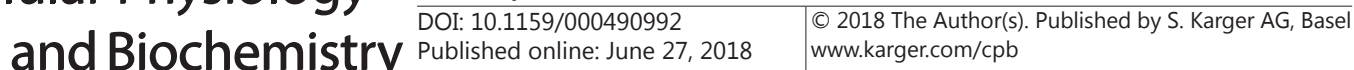 \\ Wu et al.: Transcriptomic Analysis of LSCC Stem Cells}

2015021198, 201601D011087), Shanxi Province Scientific and Technological Achievements Transformation Guidance Foundation (201604D131002, 201604D132040), Key Technology Innovation Platform Foundation for Head and Neck Cancer Research of Shanxi Province (201605D151003), Scientific and Technological Innovation Programs of Higher Education Institutions in Shanxi (STIP, 2016-92, 2016-93), the Research Project of Shanxi Province Health and Family Planning Commission (201301073, 2014028, 201601037, 201601038), the Excellent talent science and technology innovation project of Shanxi Province (201605D211029,201705D211018), InnovationFundforGraduateStudentofShanxi Province (2016-119, 2016-101), Outstanding Youth Development Foundation of The First Hospital Affiliated with Shanxi Medical University (YR1601), Youth Foundation of The First Hospital Affiliated with Shanxi Medical University (YQ1503), Startup Foundation for Doctors of Shanxi Medical University (BS03201624), and Startup Foundation for Doctors of Liaoning Province (201601355).

\section{Disclosure Statement}

The authors declare that they have no conflicts of interest.

\section{References}

$>1$ Steuer CE, El-Deiry M, Parks JR, Higgins KA, Saba NF: An update on larynx cancer. CA Cancer J Clin 2017;67:31-50.

-2 Shah PK, Shah KK, Karakousis GC, Reinke CE, Kelz RR, Fraker DL: Regional recurrence after lymphadenectomy for clinically evident lymph node metastases from papillary thyroid cancer: a cohort study. Ann Surg Oncol 2012;19:1453-1459.

-3 Baek SK, Jung KY, Kang SM, Kwon SY, Woo JS, Cho SH, Chung EJ: Clinical risk factors associated with cervical lymph node recurrence in papillary thyroid carcinoma. Thyroid 2010;20:147-152.

4 Li Y, Atkinson K, Zhang T: Combination of chemotherapy and cancer stem cell targeting agents: Preclinical and clinical studies. Cancer Lett 2017;396:103-109.

5 Pearson AT, Jackson TL, Nör JE: Modeling head and neck cancer stem cell-mediated tumorigenesis. Cell Mol Life Sci 2016;73:3279-3289.

6 Wang J, Wu Y, Gao W, Li F, Bo Y, Zhu M, Fu R, Liu Q, Wen S, Wang B: Identification and characterization of CD133+CD44+ cancer stem cells from human laryngeal squamous cell carcinoma cell lines. J Cancer 2017;8:497-506.

7 Bartel DP: MicroRNAs: target recognition and regulatory functions. Cell 2009;136:215-233.

8 Memczak S, Jens M, Elefsinioti A, Torti F, Krueger J, Rybak A, Maier L, Mackowiak SD, Gregersen LH, Munschauer M, Loewer A, Ziebold U, Landthaler M, Kocks C, le Noble F, Rajewsky N: Circular RNAs are a large class of animal RNAs with regulatory potency. Nature 2013;495:333-338.

-9 Peng R, Liu Y, Cai Z, Shen F, Chen J, Hou R, Zou F: Characterization and Analysis of Whole Transcriptome of Giant Panda Spleens: Implying Critical Roles of Long Non-Coding RNAs in Immunity. Cell Physiol Biochem 2018;46:1065-1077.

10 St LG, Wahlestedt C, Kapranov P: The Landscape of long noncoding RNA classification. Trends Genet 2015;31:239-251.

11 Pertea M, Kim D, Pertea GM, Leek JT, Salzberg SL: Transcript-level expression analysis of RNA-seq experiments with HISAT, StringTie and Ballgown. Nat Protoc 2016;11:1650-1667.

12 Trapnell C, Williams BA, Pertea G, Mortazavi A, Kwan G, van Baren MJ, Salzberg SL, Wold BJ, Pachter L: Transcript assembly and quantification by RNA-Seq reveals unannotated transcripts and isoform switching during cell differentiation. Nat Biotechnol 2010;28:511-515.

13 Kozomara A, Griffiths-Jones S: miRBase: annotating high confidence microRNAs using deep sequencing data. Nucleic Acids Res 2014;42(Database issue):D68-73. 


\section{Cellular Physiology Cell Physiol Biochem 2018;47:1696-1710 \begin{tabular}{l|l} 
DOI: 10.1159/000490992 & O 2018 The Author(s). Published by S. Karger AG, Basel \\
www.karger.com/cpb
\end{tabular}}

Wu et al.: Transcriptomic Analysis of LSCC Stem Cells

14 Wang L, Feng Z, Wang X, Wang X, Zhang X: DEGseq: an R package for identifying differentially expressed genes from RNA-seq data. Bioinformatics 2010;26:136-138.

15 Glažar P, Papavasileiou P, Rajewsky N: circBase: a database for circular RNAs. RNA 2014;20:1666-1670.

-16 Huang dW, Sherman BT, Lempicki RA: Systematic and integrative analysis of large gene lists using DAVID bioinformatics resources. Nat Protoc 2009;4:44-57.

17 Smoot ME, Ono K, Ruscheinski J, Wang PL, Ideker T: Cytoscape 2.8: new features for data integration and network visualization. Bioinformatics 2011;27:431-432.

18 Lacroix M: Persistent use of “false" cell lines. Int J Cancer 2008;122:1-4.

-19 Song YZ, Li JF: Circular RNA hsa_circ_0001564 regulates osteosarcoma proliferation and apoptosis by acting miRNA sponge. Biochem Biophys Res Commun 2018;495:2369-2375.

20 Shukla G, Khera HK, Srivastava AK, Khare P, Patidar R, Saxena R: Therapeutic Potential, Challenges and Future Perspective of Cancer Stem Cells in Translational Oncology: A Critical Review. Curr Stem Cell Res Ther 2017;12:207-224.

21 Wan G, Zhou L, Xie M, Chen H, Tian J: Characterization of side population cells from laryngeal cancer cell lines. Head Neck 2010;32:1302-1309.

-22 Tabor MH, Clay MR, Owen JH, Bradford CR, Carey TE, Wolf GT, Prince ME: Head and neck cancer stem cells: the side population. Laryngoscope 2011;121:527-533.

23 Peng Q, Qin J, Zhang Y, Cheng X, Wang X, Lu W, Xie X, Zhang S: Autophagy maintains the stemness of ovarian cancer stem cells by FOXA2 J Exp Clin Cancer Res 2017;36:171.

24 Aschacher T, Wolf B, Enzmann F, Kienzl P, Messner B, Sampl S, Svoboda M, Mechtcheriakova D, Holzmann $\mathrm{K}$, Bergmann M: LINE-1 induces hTERT and ensures telomere maintenance in tumour cell lines. Oncogene 2016;35:94-104.

25 Xiong Q Feng J, Li ST, Zhang GY, Qiao ZX, Chen Z, Wu Y, Lin Y, Li T, Ge F, Zhao JD: Integrated transcriptomic and proteomic analysis of the global response of Synechococcus to high light stress. Mol Cell Proteomics 2015;14:1038-1053.

-26 Lin Z, An J, Wang J, Niu J, Ma C, Wang L, Yuan G, Shi L, Liu L, Zhang J, Zhang Z, Qi J, Lin S: Integrated analysis of 454 and Illumina transcriptomic sequencing characterizes carbon flux and energy source for fatty acid synthesis in developing Lindera glauca fruits for woody biodiesel. Biotechnol Biofuels 2017;10:134.

27 Yan Y, Tsukamoto O, Nakano A, Kato H, Kioka H, Ito N, Higo S, Yamazaki S, Shintani Y, Matsuoka K, Liao Y, Asanuma H, Asakura M, Takafuji K, Minamino T, Asano Y, Kitakaze M, Takashima S: Augmented AMPK activity inhibits cell migration by phosphorylating the novel substrate Pdlim5 Nat Commun 2015;6:6137.

28 Li Y, Gao Y, Xu Y, Sun X, Song X, Ma H, Yang M: si-RNA-mediated knockdown of PDLIM5 suppresses gastric cancer cell proliferation in vitro. Chem Biol Drug Des 2015;85:447-453.

29 Han N, Zhao W, Zhang Z, Zheng P: MiR-328 suppresses the survival of esophageal cancer cells by targeting PLCE1 Biochem Biophys Res Commun 2016;470:175-180.

30 Ma W, Ma CN, Zhou NN, Li XD, Zhang YJ: Up- regulation of miR-328-3p sensitizes non-small cell lung cancer to radiotherapy. Sci Rep 2016;6:31651.

-31 Xu XT, Xu Q, Tong JL, Zhu MM, Nie F, Chen X, Xiao SD, Ran ZH: MicroRNA expression profiling identifies miR328 regulates cancer stem cell-like SP cells in colorectal cancer. Br J Cancer 2012;106:1320-1330.

-32 Wang Y, Kelber JA, Tran Cao HS, Cantin GT, Lin R, Wang W, Kaushal S, Bristow JM, Edgington TS, Hoffman RM, Bouvet M, Yates JR, Klemke RL: Pseudopodium-enriched atypical kinase 1 regulates the cytoskeleton and cancer progression [corrected]. Proc Natl Acad Sci U S A 2010;107:10920-10925.

-33 Kazi JU, Rupar K, Marhäll A, Moharram SA, Khanum F, Shah K, Gazi M, Nagaraj SR, Sun J, Chougule RA, Rönnstrand L: ABL2 suppresses FLT3-ITD-induced cell proliferation through negative regulation of AKT signaling. Oncotarget 2017;8:12194-12202.

-34 Qiang XF, Zhang ZW, Liu Q, Sun N, Pan LL, Shen J, Li T, Yun C, Li H, Shi LH: miR-20a promotes prostate cancer invasion and migration through targeting ABL2 J Cell Biochem 2014;115:1269-1276.

35 Hill VK, Dunwell TL, Catchpoole D, Krex D, Brini AT, Griffiths M, Craddock C, Maher ER, Latif F: Frequent epigenetic inactivation of KIBRA, an upstream member of the Salvador/Warts/Hippo (SWH) tumor suppressor network, is associated with specific genetic event in B-cell acute lymphocytic leukemia. Epigenetics 2011;6:326-332.

-36 Yu J, Zheng Y, Dong J, Klusza S, Deng WM, Pan D: Kibra functions as a tumor suppressor protein that regulates Hippo signaling in conjunction with Merlin and Expanded. Dev Cell 2010;18:288-299. 


\section{Cellular Physiology Cell Physiol Biochem 2018;47:1696-1710 \begin{tabular}{l|l} 
DOI: 10.1159/000490992 \\
and Biochemistry
\end{tabular} $\begin{aligned} & \text { Published 2018 The Author(s). Published by S. Karger AG, Basel } \\
& \text { www.karger.com/cpb }\end{aligned}$ \\ Wu et al.: Transcriptomic Analysis of LSCC Stem Cells}

37 Gafar AA, Draz HM, Goldberg AA, Bashandy MA, Bakry S, Khalifa MA, AbuShair W, Titorenko VI, Sanderson JT: Lithocholic acid induces endoplasmic reticulum stress, autophagy and mitochondrial dysfunction in human prostate cancer cells. PeerJ 2016;4:e2445.

-38 Cubillos-Ruiz JR, Bettigole SE, Glimcher LH: Tumorigenic and Immunosuppressive Effects of Endoplasmic Reticulum Stress in Cancer. Cell 2017;168:692-706.

-39 Ménétret JF, Hegde RS, Heinrich SU, Chandramouli P, Ludtke SJ, Rapoport TA, Akey CW: Architecture of the ribosome-channel complex derived from native membranes. J Mol Biol 2005;348:445-457.

40 Mesbah K, Camus A, Babinet C, Barra J: Mutation in the Trapalpha/Ssr1 gene, encoding transloconassociated protein alpha, results in outflow tract morphogenetic defects. Mol Cell Biol 2006;26:7760-7771.

-41 Pekarsky Y, Balatti V, Palamarchuk A, Rizzotto L, Veneziano D, Nigita G, Rassenti LZ, Pass HI, Kipps TJ, Liu CG, Croce CM: Dysregulation of a family of short noncoding RNAs, tsRNAs, in human cancer. Proc Natl Acad Sci U S A 2016;113:5071-5076.

42 Yamaguchi N, Osaki M, Onuma K, Yumioka T, Iwamoto H, Sejima T, Kugoh H, Takenaka A, Okada F: Identification of MicroRNAs Involved in Resistance to Sunitinib in Renal Cell Carcinoma Cells. Anticancer Res 2017;37:2985-2992.

43 Bouvard V, Baan R, Straif K, Grosse Y, Secretan B, El Ghissassi F, Benbrahim-Tallaa L, Guha N, Freeman C, Galichet L, Cogliano V: A review of human carcinogens--Part B: biological agents. Lancet Oncol 2009;10:321-322.

44 Morshed K: Association between human papillomavirus infection and laryngeal squamous cell carcinoma. J Med Virol 2010;82:1017-1023.

45 Talati PG, Gu L, Ellsworth EM, Girondo MA, Trerotola M, Hoang DT, Leiby B, Dagvadorj A, McCue PA, Lallas CD, Trabulsi EJ, Gomella L, Aplin AE, Languino L, Fatatis A, Rui H, Nevalainen MT: Jak2-Stat5a/b Signaling Induces Epithelial-to-Mesenchymal Transition and Stem-Like Cell Properties in Prostate Cancer. Am J Pathol 2015;185:2505-2522. 